\title{
Combination of thrombin-antithrombin complex, plasminogen activator inhibitor-1, and protein $C$ activity for early identification of severe coagulopathy in initial phase of sepsis: a prospective observational study
}

Kansuke Koyama ${ }^{1,2^{*}}$, Seiji Madoiwa ${ }^{1,2^{*}}$, Shin Nunomiya' ${ }^{1}$ Toshitaka Koinuma', Masahiko Wada ${ }^{1}$, Asuka Sakata ${ }^{2}$, Tsukasa Ohmori ${ }^{2}$, Jun Mimuro ${ }^{2}$ and Yoichi Sakata ${ }^{2}$

\begin{abstract}
Introduction: Current criteria for early diagnosis of coagulopathy in sepsis are limited. We postulated that coagulopathy is already complicated with sepsis in the initial phase, and severe coagulopathy or disseminated intravascular coagulation (DIC) becomes overt after progressive consumption of platelet and coagulation factors. To determine early diagnostic markers for severe coagulopathy, we evaluated plasma biomarkers for association with subsequent development of overt DIC in patients with sepsis.
\end{abstract}

Methods: A single-center, prospective observational study was conducted in an adult ICU at a university hospital. Plasma samples were obtained from patients with sepsis at ICU admission. Fourteen biomarkers including global markers (platelet count, prothrombin time, activated partial thromboplastin time, fibrinogen and fibrin degradation product (FDP)); markers of thrombin generation (thrombin-antithrombin complex (TAT) and soluble fibrin); markers of anticoagulants (protein $C(P C)$ and antithrombin); markers of fibrinolysis (plasminogen, $a_{2}$-plasmin inhibitor (PI), plasmin- $\mathrm{a}_{2}-\mathrm{PI}$ complex, and plasminogen activator inhibitor (PAI)-1); and a marker of endothelial activation (soluble E-selectin) were assayed. Patients who had overt DIC at baseline were excluded, and the remaining patients were followed for development of overt DIC in 5 days, and for mortality in 28 days.

Results: A total of 77 patients were enrolled, and 37 developed overt DIC within the following 5 days. Most patients demonstrated hemostatic abnormalities at baseline with 98.7\% TAT, 97.4\% FDP and 88.3\% PC. Most hemostatic biomarkers at baseline were significantly associated with subsequent development of overt DIC. Notably, TAT, PAI-1 and PC discriminated well between patients with and without developing overt DIC (area under the receiver operating characteristic curve (AUROC), 0.77 (95\% confidence interval, 0.64 to 0.86 ); 0.87 (0.78 to 0.92 ); 0.85 (0.76 to 0.91 ), respectively), and using the three together, significantly improved the AUROC up to 0.95 (vs. TAT, PAI-1, and PC). Among the significant diagnostic markers for overt DIC, TAT and PAI-1 were also good predictors of 28-day mortality (AUROC, 0.77 and 0.81 , respectively).

(Continued on next page)

\footnotetext{
* Correspondence: k_koyama@jichi.ac.jp; madochan@ms2.jichi.ac.jp 'Division of Intensive Care, Department of Anesthesiology \& Intensive Care Medicine, Jichi Medical University School of Medicine, 3311-1 Yakushiji, Shimotsuke, Tochigi 329-0498, Japan

${ }^{2}$ Research Divisions of Cell and Molecular Medicine, Center of Molecular Medicine, Jichi Medical University School of Medicine, 3311-1 Yakushiji, Shimotsuke, Tochigi 329-0498, Japan
}

\section{Biomed Central}

(c) 2014 Koyama et al.; licensee BioMed Central Ltd. This is an Open Access article distributed under the terms of the Creative Commons Attribution License (http://creativecommons.org/licenses/by/2.0), which permits unrestricted use, distribution, and reproduction in any medium, provided the original work is properly cited. The Creative Commons Public Domain Dedication waiver (http://creativecommons.org/publicdomain/zero/1.0/) applies to the data made available in this article, unless otherwise stated. 
(Continued from previous page)

Conclusions: Severe coagulation and fibrinolytic abnormalities on ICU admission were associated with subsequent development of overt DIC. A single measurement of TAT, PAI-1, and PC activity could identify patients with ongoing severe coagulopathy, early in the course of sepsis.

\section{Introduction}

Sepsis is frequently complicated with coagulopathy [1]. The severity of sepsis-associated coagulopathy is variable, ranging from subclinical abnormalities that are detectable by a mild decrease in platelet count and prolongation of global clotting times, to severe forms of coagulopathy or disseminated intravascular coagulation (DIC) [2]. The incidence of DIC is up to 25 to $50 \%$ in patients with sepsis [3].

Septic DIC is characterized by systemic intravascular activation of coagulation, and microvascular endothelial injury with impaired anticoagulation and insufficient fibrinolysis, which leads to widespread thrombosis in microvasculature. In sepsis, DIC has a feature of vascular endothelial dysfunction, as well as being an etiological factor in the failure of other organs: excessive thrombin generation and subsequent fibrin deposition exacerbate inflammation and ischemia, contributing to organ damage [4]. A number of studies have reported that DIC is an independent risk factor for organ dysfunction and mortality in patients with sepsis $[2,3,5]$. DIC might, therefore, be an important therapeutic target in the management of sepsis, and the development of reliable methods for early identification of DIC is a high priority.

However, the early diagnosis of sepsis-associated coagulopathy and evaluation of its severity is still challenging [6]. Currently, the overt DIC criteria of the International Society on Thrombosis and Haemostasis (ISTH) are the diagnostic standard for severe coagulopathy in sepsis [4]. Although the ISTH criteria for overt DIC are simple and widely used, and shown to be associated with organ failure and mortality, they have limited application in the early phase of sepsis to improve outcome $[7,8]$. The ISTH overt DIC criteria use global markers, such as prothrombin time (PT) and platelet count for scoring. The coagulation factors and platelets are consumed and decrease over time because of progressive thrombin generation and endothelial injury, thus it takes several days to reveal their abnormalities and fulfill the overt DIC criteria in the course of sepsis $[9,10]$. Furthermore, introduction of the concept of pre-DIC, which is considered as the stage prior to overt DIC, has failed to predict disease progression. An ISTH subcommittee defined non-overt DIC as compensated coagulopathy, or pre-stage DIC, for the early diagnosis of overt DIC [4]. However, previous studies have shown that only 10 to $30 \%$ of patients with non-overt DIC progressed to overt
DIC, although the mortality rates were similar between patients with non-overt and overt DIC $[6,11]$.

In the past decade, there has been increasing evidence that inflammation and coagulation play pivotal roles in the pathogenesis of sepsis $[12,13]$. Pro-inflammatory cytokines produced by the host response against infection stimulate tissue factor expression and lead to activation of coagulation. An activated coagulation system in turn modulates inflammatory activity through specific receptors, such as protease-activated receptors. Considering that excessive crosstalk between inflammation and coagulation is ongoing from the onset of sepsis, severe coagulopathy may have developed early in the course.

The objective of this study was to identify hemostatic biomarkers that can be used for early diagnosis of severe coagulopathy in patients with sepsis. We postulate that severe coagulopathy has already developed in the initial phase of sepsis, and is related to the subsequent fulfillment of the criteria for overt DIC [14]. We, therefore, evaluated the association between plasma biomarkers measured at the time of intensive care unit (ICU) admission and development of overt DIC in the following five days. We also investigated the hemostatic biomarkers as predictors for 28-day mortality.

\section{Material and methods}

\section{Study design and setting}

This was a single-center, prospective observational study, that was conducted in a 12-bed medicosurgical ICU at a university hospital from January 2012 to June 2013. The study was approved by the Institutional Research Ethics Committee of Jichi Medical University, and informed consent was obtained from the patients or their families.

The consecutive patients who were admitted to the ICU because of sepsis, and without overt DIC on ISTH criteria at the time of ICU admission, were enrolled. Sepsis was defined according to the 2001 International Sepsis Definitions Conference [15]. Exclusion criteria were: age $<18$ years, presence of decompensated cirrhosis (Child-Pugh class B or $\mathrm{C}$ ), hematological disorders, chronic renal failure on hemodialysis, and history of therapeutic anticoagulation or blood transfusion during the preceding four weeks.

Clinical and demographic data, including age, sex, comorbidity and Acute Physiology and Chronic Health Evaluation (APACHE) II scores [16], were recorded on ICU admission. Sequential Organ Failure Assessment (SOFA) score [17] except for coagulation (platelet count), 
and overt DIC score on ISTH criteria were determined daily. ISTH non-overt DIC score, and acute DIC score established by the Japanese Association for Acute Medicine (JAAM) [18] were also calculated daily as early diagnostic systems for DIC.

The primary endpoint was the development of overt DIC within the first five days of ICU stay. A score $\geq 5$ on the ISTH criteria was defined as overt DIC. The secondary endpoint was 28-day all-cause mortality. Plasma samples were drawn from the eligible patients within $6 \mathrm{~h}$ of ICU admission, and the patients were followed for 5 days for overt DIC score and 28 days for mortality.

\section{Biomarker measurements}

Plasma biomarkers were measured at the time of ICU admission (Day 0) as baseline, and on days 1 to 3 . We classified 14 biomarkers into five categories: global markers (platelet count, prothrombin time (PT) and PT-international normalized ratio (PT-INR), activated partial thromboplastin time, fibrinogen, fibrin degradation product (FDP)); markers of thrombin generation (thrombin-antithrombin complex (TAT), soluble fibrin (SF)); markers of anticoagulants activity (protein $\mathrm{C}(\mathrm{PC})$, antithrombin (AT)); markers of fibrinolytic activity (plasminogen, $\alpha_{2}$-plasmin inhibitor (PI), plasminogen activator inhibitor (PAI)-1, plasmin- $\alpha_{2}$-PI complex (PIC)); and a marker of endothelial activation (soluble E-selectin (sES)).

Blood samples were collected heparin-free and centrifuged at 2,500 rpm at $4^{\circ} \mathrm{C}$ in citrated tubes. Global markers, TAT, PC, AT, plasminogen, $\alpha_{2}$-PI and PIC were assayed using the CS-2100i automatic coagulation analyzer (Sysmex, Hyogo, Japan) immediately after the samples were collected. Berichrom assays (Siemens Healthcare Diagnostics, Tokyo, Japan) were used for PC, AT, plasminogen and $\alpha 2$-PI activities, and TAT/PIC test $\mathrm{F}$ enzyme immunoassay (Sysmex) were used for measurements of TAT and PIC levels, respectively. SF, PAI-1 and E-selectin were measured with the stored samples, which were frozen at $-80^{\circ} \mathrm{C}$ within $2 \mathrm{~h}$ of collection, using iatroSF, tPAI test and sES latex photometric immunoassay, respectively (Mitsubishi Chemical Medience, Tokyo, Japan).

\section{Patient management}

Our facility provides 24-h coverage by attending ICU physicians. Management of patients followed the Surviving Sepsis Campaign Guidelines (SSCG) with the goal of initial resuscitation and infection control [19]. Patients received mechanical prophylactic treatment without concomitant low-dose heparin, until no active bleeding or severe coagulopathy was confirmed. Antithrombin substitution therapy was at the discretion of the ICU physicians, limited for the patients with AT activity $<50 \%$ after the plasma samples at baseline were collected. The patients with bleeding risk or complications were transfused with platelet concentrate or fresh frozen plasma as decided by the ICU physicians.

\section{Data analysis}

The study population was grouped according to the development of overt DIC. Statistical differences between the groups were analyzed using Wilcoxon rank-sum test for non-normally distributed variables, and the $x^{2}$, or Fisher's exact test for categorical variables as appropriate. Biomarker abnormalities were defined as values higher than the upper limit of normal, or lower than the lower limit of normal, which were used in practice at our institution. Receiver operating characteristic (ROC) curve analysis was performed to calculate the area under the receiver operating characteristic curve (AUROC) of the 14 biomarkers at baseline for the development of overt DIC, and of those at baseline and at Day 2 for 28-day mortality. The AUROC for APACHE II score and pre-DIC scores (by ISTH non-overt DIC, and JAAM acute DIC criteria) at baseline were also calculated for comparison. The best cutoff values were calculated to maximize the sum of sensitivity and specificity. Positive predictive value (PPV) and negative predictive value (NPV) were also calculated. To assess the bivariable association among biomarkers, Spearman rank correlation coefficients ( $r$ value) along with the associated $P$-value were calculated, and $r<0.5$ was considered as no evidence of collinearity. A multivariate logistic regression model based on a forward stepwise method was used to identify the best combination to discriminate the development of overt DIC. To assess the impact of biomarkers on survival, Kaplan-Meier estimates were used to illustrate trends in 28-day mortality and the log-rank test was performed. All $P$-values were two-tailed, and $P<0.05$ was considered statistically significant. Data were analyzed using JMP version 10 (SAS Institute, Tokyo, Japan).

\section{Results}

\section{Patient characteristics and outcomes}

One hundred, eleven patients were admitted to the ICU because of sepsis during the study period. Thirty-four patients were excluded according to the study criteria, and the remaining 77 patients were enrolled. The baseline characteristics and prognosis of the study population are described in Table 1. Of 77 patients with sepsis, 37 (48.1\%) developed overt DIC within five days of their ICU stay. Patients who newly developed overt DIC were more severely ill with a higher APACHE II score, maximum SOFA scores and 28-day mortality, compared with patients who did not develop overt DIC. No therapeutic heparin was administered during the study period. Prophylactic low-dose heparin was used more frequently in patients without DIC than in those who developed overt DIC (50.0 vs. $10.8 \%, P=0.0001$ ). Platelet concentrate, fresh frozen plasma and antithrombin were more frequently 
Table 1 Baseline characteristics and outcomes of the 77 patients with sepsis

\begin{tabular}{|c|c|c|c|c|}
\hline & All patients $(n=77)$ & Develop DIC $(n=37)$ & No DIC $(n=40)$ & $P$-value* \\
\hline \multicolumn{5}{|l|}{ Demographics } \\
\hline Age (years) & $69.9 \pm 12.9$ & $70.7 \pm 13.2$ & $69.1 \pm 12.7$ & 0.58 \\
\hline Male & $42(54.5)$ & $16(43.2)$ & $26(65.0)$ & 0.069 \\
\hline \multicolumn{5}{|l|}{ Source of sepsis } \\
\hline Pulmonary infection & 15 (19.5) & $7(18.9)$ & $8(20.0)$ & 0.91 \\
\hline Abdominal infection & $43(55.8)$ & $22(59.5)$ & $21(52.5)$ & 0.54 \\
\hline Urinary tract infection & $5(6.5)$ & $3(8.1)$ & $2(5.0)$ & 0.58 \\
\hline Soft tissue infection & $11(14.3)$ & $3(8.1)$ & $8(20.0)$ & 0.13 \\
\hline Blood stream infection & $2(2.6)$ & $2(5.4)$ & $0(0.0)$ & 0.084 \\
\hline \multicolumn{5}{|l|}{ Comorbidities } \\
\hline IHD & $7(9.1)$ & $2(5.4)$ & $5(12.5)$ & 0.27 \\
\hline $\mathrm{CHF}$ & $2(2.6)$ & $0(0.0)$ & $2(5.0)$ & 0.11 \\
\hline Arrhythmia & $3(3.9)$ & $3(8.1)$ & $0(0.0)$ & 0.033 \\
\hline COPD & $6(7.8)$ & $1(2.7)$ & $5(12.5)$ & 0.094 \\
\hline CKD & $10(13.0)$ & $6(16.2)$ & $4(10.0)$ & 0.42 \\
\hline CVD & $3(3.9)$ & $2(5.4)$ & $1(2.5)$ & 0.51 \\
\hline \multicolumn{5}{|l|}{ Severity of illness } \\
\hline APACHE II score & $25.4 \pm 7.9$ & $28.8 \pm 8.2$ & $22.2 \pm 6.1$ & 0.0002 \\
\hline \multicolumn{5}{|c|}{ Organ dysfunction (days 0 to 5 ) } \\
\hline $\max$ SOFA score ${ }^{* *}$ & $9(7$ to 11$)$ & 10 (9 to 14$)$ & 7 (4 to 9) & 0.0001 \\
\hline \multicolumn{5}{|l|}{ Prognosis } \\
\hline ICU-free days & $18(10$ to 21$)$ & $16(0$ to 19$)$ & 21 (17 to 23$)$ & 0.0001 \\
\hline 28-day mortality & $15(19.5)$ & $13(35.1)$ & $2(5.0)$ & 0.0005 \\
\hline
\end{tabular}

Data are expressed as mean \pm SD, median (interquartile range), or No. (\%).

APACHE, acute physiology and chronic health evaluation; CHF, chronic heart failure; CKD, chronic kidney disease; COPD, chronic obstructive pulmonary disease; CVD, cerebrovascular disease; IHD, ischemic heart disease.

*Comparison of groups with and without subsequent development of overt DIC.

**maximum SOFA scores except for score of coagulation (platelet count) during the first five days of ICU stay.

administered to patients who developed overt DIC than to those who did not $(24.3$ vs. $0 \%, P=0.0001 ; 29.7$ vs. $7.5 \%$, $P=0.012 ; 56.8$ vs. $5.0 \%, P<0.0001$, respectively).

\section{Evidence of biomarker abnormalities at baseline and subsequent changes over time}

The majority of the 77 patients with sepsis presented with plasma biomarker abnormalities at the time of admission (Day 0), as indicated by elevated TAT $(98.7 \%$ of patients) and FDP (97.4\% of patients), and decreased activity of PC (88.3\% of patients, Table 2$)$. In contrast, decreased platelet count, prolonged PT-INR or decreased level of fibrinogen was not frequently observed on Day 0 in patients with sepsis.

Plasma biomarkers of platelet, PT-INR, FDP, TAT, PAI-1 and $\mathrm{PC}$ over time (days 0 to 3 ) in patients with and without subsequent development of overt DIC are shown in Figure 1 (other studied biomarkers are shown in Additional file 1). On Day 0, there were marked increases in TAT and PAI-1, and decreases in PC, plasminogen and $\alpha_{2}$-PI activities, which were particularly marked in patients with subsequent development of overt DIC. Notably, TAT and PAI-1 were the highest on Day 0 and gradually returned to normal in patients who developed overt DIC (TAT on Day 0 vs. Day 2, $P=0.013$; PAI-1, $P=0.0035)$, whereas platelet count and PT-INR were around the normal range on Day 0 and exacerbated until days 2 to 3 (platelet on Day 0 vs. Day 2, $P<0.0001$; PT-INR, $P=0.0043$ ).

\section{Discrimination capacity of plasma biomarkers at baseline for subsequent development of overt DIC}

We conducted ROC curve analysis to evaluate the ability of biomarkers to discriminate among patients who subsequently developed overt DIC and those who did not. The AUROCs and best calculated cutoff values, PPV and NPV, are shown in Table 3. The AUROCs and PPVs for the development of overt DIC were high for TAT, PC, AT, plasminogen, $\alpha 2-\mathrm{PI}$ and PAI-1. For the comparison between discrimination abilities of plasma biomarkers and those of severity of illness, and pre-DIC scores at 
Table 2 Plasma biomarkers at baseline (Day 0) in patients with sepsis

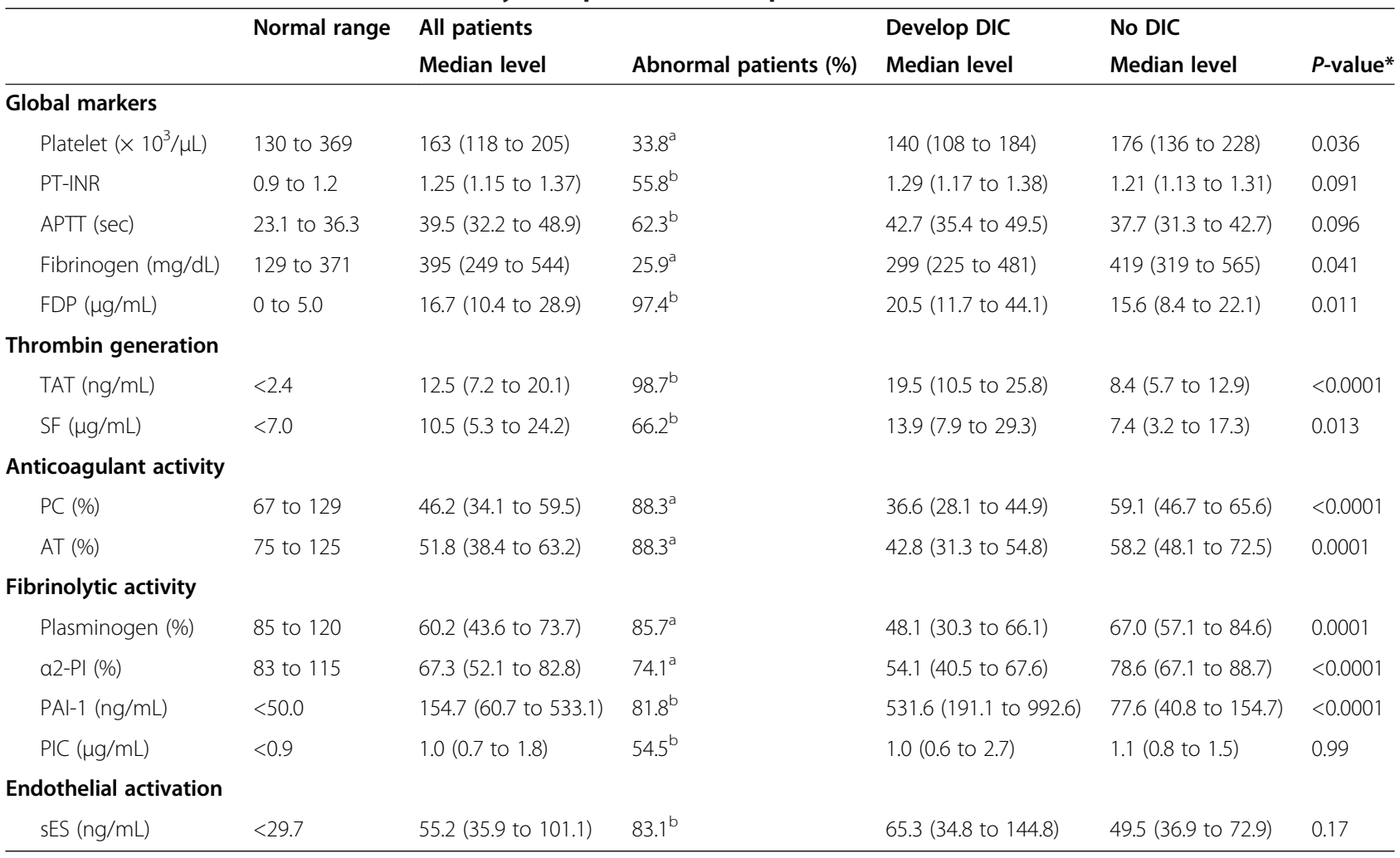

a2-PI, a2-plasmin inhibitor activity; APTT, activated partial thromboplastin time; AT, antithrombin activity; DIC, disseminated intravascular coagulation; FDP, fibrin degradation products; PAI-1, plasminogen activator inhibitor-1; PC, protein C activity; PIC, plasmin-a2-plasmin inhibitor complex; PT-INR, prothrombin time-international normalized ratio; sES, soluble E-selectin; SF, soluble fibrin; TAT, thrombin-antithrombin complex.

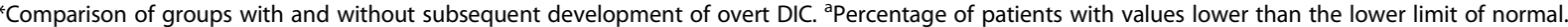

bercentage of patients with values higher than the upper limit of normal.

baseline, the AUROCs and PPVs were also calculated for APACHE II scores (AUROC, 0.72, (95\% confidence interval, 0.61 to 0.82$)$; PPV, 0.62), ISTH non-overt DIC scores (AUROC, 0.71 (0.59 to 0.80); PPV, 0.58), and JAAM DIC scores (AUROC, 0.68 (0.55 to 0.78); PPV, 0.62) with relatively low PPV values.

\section{Correlation and multivariate analysis to identify significant diagnostic biomarkers for subsequent development of overt DIC}

To identify efficient diagnostic markers for the development of overt DIC, we undertook further analysis of significant biomarkers with AUROC $>0.7$ and PPV $>0.7$, which were superior to the results of APACHE II scores or pre-DIC scores. First, we calculated Spearman rank correlation coefficients for TAT, PC, AT, plasminogen, $\alpha_{2}$ PI and PAI-1 to rule out collinearity among the significant biomarkers. We found a strong and significant correlation with $r>0.5$ between each pair of PC, AT, plasminogen and $\alpha_{2}$-PI values (Additional file 2). However, TAT and PAI-1 were not so highly correlated with PC.

Next, we conducted a multivariate stepwise logistic regression analysis, and found that TAT, PAI-1 and PC were the best combination to discriminate between patients with and without development of overt DIC. These three biomarkers remained significantly associated with overt DIC, even after adjustment for APACHE II score in separate models (TAT, $P=0.0002$; PAI- $1, P=0.0001$; PC, $P<0.0001$, respectively). Furthermore, the combination of TAT, PAI-1 and PC substantially improved discrimination of the development of overt DIC, compared with each marker alone (AUROC 0.95 (vs. TAT, $P=0.0004$; vs. PAI-1, $P=0.033$; vs. PC, $P=0.025)$, Figure 2 ).

\section{Plasma biomarkers on days 0 and 2 as predictors of 28-day mortality}

Univariate analysis revealed that only TAT and PAI-1 at baseline were significant predictors of 28-day mortality among the biomarkers that had good discriminative power for the development of overt DIC (Table 4 and Additional file 3). Based on the best calculated cutoff values, cutoff points at baseline were set at $18 \mathrm{ng} / \mathrm{mL}$ for TAT and $270 \mathrm{ng} / \mathrm{mL}$ for PAI-1. The Kaplan-Meier survival curve for patients with sepsis demonstrated that TAT $>18 \mathrm{ng} / \mathrm{mL}$ and/or PAI-1 $>270 \mathrm{ng} / \mathrm{mL}$ on admission were significantly correlated with higher mortality $(P=0.0024$, Figure 3$)$.

Most of the studied Day 2 markers had higher AUROCs for prediction of 28-day mortality compared with 

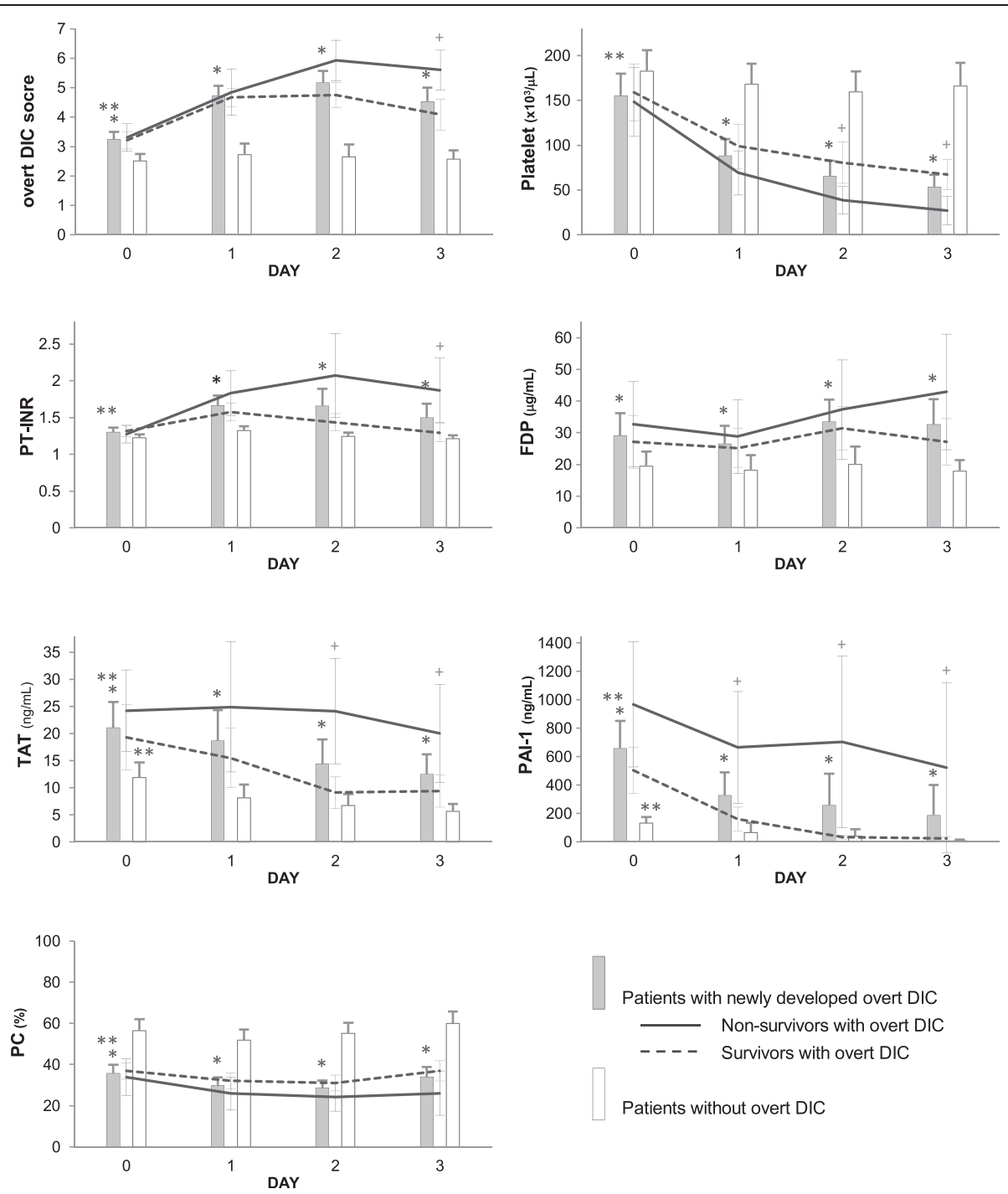

$$
\begin{array}{cl}
\text { Patients with newly developed overt DIC } \\
-\ldots \text { Non-survivors with overt DIC } \\
--- & \text { Survivors with overt DIC }
\end{array}
$$

Patients without overt DIC

Figure 1 Time course of overt DIC scores and hemostatic biomarkers from baseline to Day 3. Overt disseminated intravascular coagulation (DIC) scores, platelet count, prothrombin time-international normalized ratio (PT-INR), fibrin degradation product (FDP), thrombin-antithrombin complex (TAT), plasminogen activator inhibitor-1 (PAI-1) and protein C (PC) for patients with and without subsequent development of overt DIC (gray vs. white bars), and for survivors (dotted line) and non-survivors (solid line) among patients with overt DIC. Data are expressed as mean and $95 \% \mathrm{Cl}$. ${ }^{*} P<0.05$ between patients with and without overt DIC on the same day. ${ }^{* *} P<0.05$ between patients on Day 0 versus Day $2 .{ }^{+} P<0.05$ between survivors and non-survivors with overt DIC on the same day.

Day 0 markers (Table 4 and Additional file 3). Among the Day 2 biomarkers, TAT, SF and PAI-1 remained statistically significant for prediction of 28-day mortality after adjustment for APACHE II score $(P=0.0016$, $P<0.0001, P<0.0001$, respectively).

\section{Discussion}

The main findings of our study were as follows. 1) Coagulopathy developed in the initial phase of sepsis, and the severity of hemostatic biomarker abnormalities on the day of admission was associated with the subsequent development of overt DIC. 2) Among all the studied biomarkers, TAT, PAI-1 and PC had the best discriminative power for the patients who newly developed overt DIC. 3) However, only TAT and PAI-1 on Day 0 were significant predictors of 28-day mortality among the diagnostic biomarkers for the development of overt DIC. In contrast, Day 2 markers had higher predictive power for 28-day mortality compared with Day 0 markers, suggesting that persistence of severe coagulopathy was correlated with mortality.

Inflammation and coagulation constitute two host defense systems with complementary roles against infection [13], which means that an overwhelming systemic inflammatory reaction in sepsis is accompanied by severe coagulopathy, and both may contribute to tissue damage in the early phase of sepsis. In our study, most patients with 
Table 3 Area under the ROC curves of biomarkers at baseline for prediction of overt DIC

\begin{tabular}{|c|c|c|c|c|c|c|}
\hline Biomarkers (Day 0) & AUC $(95 \% \mathrm{Cl})$ & Cutoff values* & Sensitivity & Specificity & PPV & NPV \\
\hline \multicolumn{7}{|l|}{ Global markers } \\
\hline Platelet & 0.65 (0.51 to 0.76$)$ & $158\left(\times 10^{3} / \mu \mathrm{L}\right)$ & 0.62 & 0.65 & 0.62 & 0.65 \\
\hline PT-INR & 0.61 (0.48 to 0.73$)$ & 1.3 & 0.62 & 0.63 & 0.61 & 0.64 \\
\hline APTT & 0.61 (0.48 to 0.73$)$ & 42 (sec) & 0.54 & 0.75 & 0.67 & 0.64 \\
\hline Fibrinogen & 0.64 (0.51 to 0.76$)$ & $310(\mathrm{mg} / \mathrm{dL})$ & 0.54 & 0.78 & 0.69 & 0.65 \\
\hline FDP & 0.67 (0.54 to 0.78$)$ & $28(\mu \mathrm{g} / \mathrm{mL})$ & 0.43 & 0.88 & 0.76 & 0.63 \\
\hline \multicolumn{7}{|l|}{ Thrombin generation } \\
\hline TAT & 0.77 (0.64 to 0.86$)$ & $15(\mathrm{ng} / \mathrm{mL})$ & 0.67 & 0.85 & 0.81 & 0.72 \\
\hline SF & 0.67 (0.54 to 0.78$)$ & $7.9(\mu \mathrm{g} / \mathrm{mL})$ & 0.77 & 0.54 & 0.61 & 0.72 \\
\hline \multicolumn{7}{|l|}{ Anticoagulant activity } \\
\hline PC & 0.85 (0.76 to 0.91$)$ & $46(\%)$ & 0.81 & 0.79 & 0.79 & 0.82 \\
\hline AT & 0.76 (0.63 to 0.85$)$ & $46(\%)$ & 0.60 & 0.85 & 0.78 & 0.69 \\
\hline \multicolumn{7}{|l|}{ Fibrinolytic activity } \\
\hline Plasminogen & 0.76 (0.63 to 0.85$)$ & $52(\%)$ & 0.60 & 0.79 & 0.73 & 0.67 \\
\hline$a 2-P I$ & 0.79 (0.67 to 0.88$)$ & $70(\%)$ & 0.81 & 0.67 & 0.70 & 0.79 \\
\hline PAl-1 & 0.87 (0.78 to 0.92) & $269(\mathrm{ng} / \mathrm{mL})$ & 0.72 & 0.92 & 0.89 & 0.78 \\
\hline $\mathrm{PIC}$ & 0.49 (0.36 to 0.63$)$ & $1.9(\mu \mathrm{g} / \mathrm{mL})$ & 0.35 & 0.89 & 0.76 & 0.59 \\
\hline \multicolumn{7}{|l|}{ Endothelial activation } \\
\hline sES & 0.59 (0.45 to 0.72$)$ & $67(\mathrm{ng} / \mathrm{mL})$ & 0.51 & 0.72 & 0.62 & 0.61 \\
\hline
\end{tabular}

a2-PI, a2-plasmin inhibitor activity; APT, activated partial thromboplastin time; AT, antithrombin activity; AUC, area under the curve; Cl, confidence interval; FDP, fibrin degradation products; NPV, negative predictive value; PAI-1, plasminogen activator inhibitor-1; PC, protein C activity; PIC, plasmin-a2-plasmin inhibitor complex; PPV, positive predictive value; PT-INR, prothrombin time-international normalized ratio; ROC, receiver operating characteristic; sES, soluble E selectin; $\mathrm{SF}$, soluble fibrin; TAT, thrombin- antithrombin complex. ${ }^{*}$ Cutoff values were calculated to maximize the sum of sensitivity and specificity.

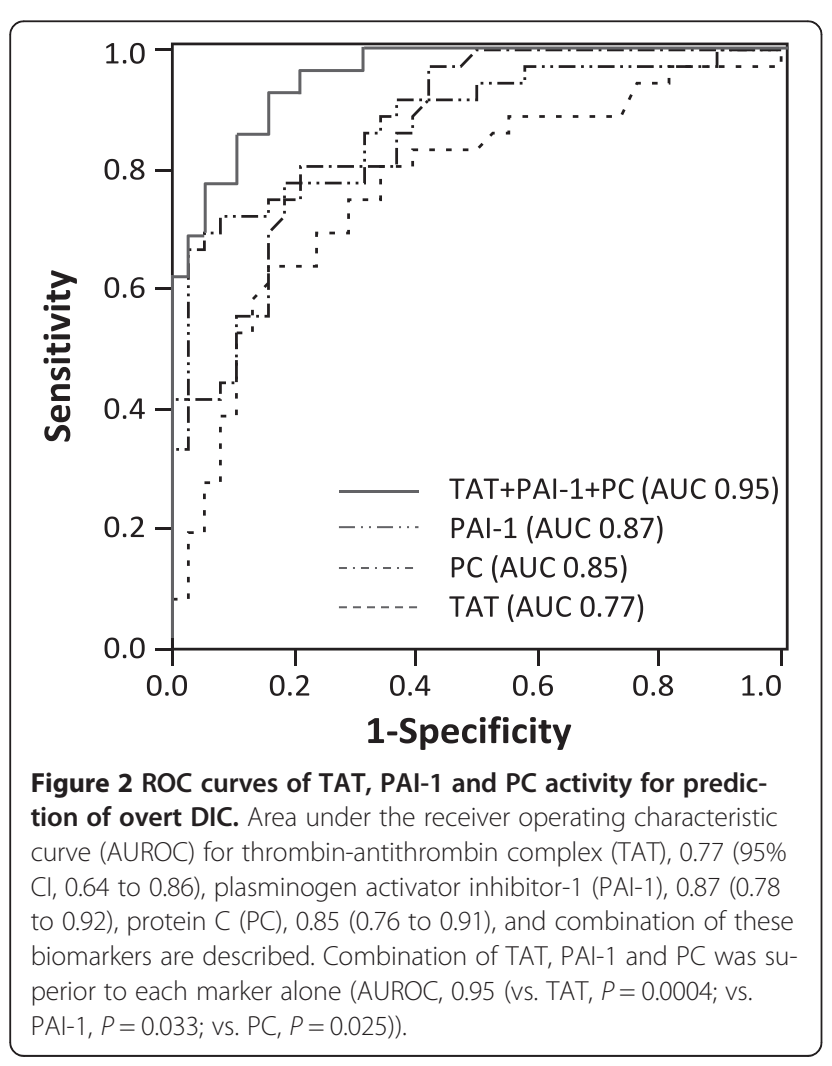

sepsis exhibited coagulation and fibrinolytic abnormalities at the time of ICU admission, which is consistent with the data from the PROWESS trial [1]. In addition, most hemostatic biomarkers measured on ICU admission were associated with subsequent fulfillment of overt DIC criteria. These results support the hypothesis that coagulopathy is present in the initial phase of sepsis, and the strategy to identify markers of acute ongoing coagulopathy, rather than to detect pre-DIC state, may be necessary for the early diagnosis of septic DIC.

The pathogenesis of DIC is primarily due to excess production of thrombin [20]. In sepsis, anticoagulation impairment and insufficient fibrinolysis also contribute to thrombin generation and fibrin deposition. Anticoagulation pathways such as the antithrombin and protein $C$ systems are impaired because of increased consumption, decreased protein synthesis, extravasation and degradation by several proteolytic enzymes such as neutrophil elastase $[21,22]$. The fibrinolytic system is largely suppressed by increased production of PAI-1, which is a principal inhibitor of this system [23,24]. In our study, increased levels of TAT and PAI-1, and decreased PC activity, were observed at the time of ICU admission and each independently discriminated the patients who developed overt DIC from those who did not. Our findings indicate that activation of coagulation, anticoagulation impairment and insufficient 
Table 4 Area under ROC curves of Day 0 and Day 2 biomarkers for prediction of mortality

\begin{tabular}{|c|c|c|c|c|c|c|c|}
\hline Biomarkers & ICU day & AUC $(95 \% \mathrm{Cl})$ & Cutoff values* & Sensitivity & Specificity & PPV & NPV \\
\hline \multicolumn{8}{|l|}{ Global markers } \\
\hline \multirow[t]{2}{*}{ Platelet } & Day 0 & 0.58 (0.41 to 0.74$)$ & $117\left(\times 10^{3} / \mu \mathrm{L}\right)$ & 0.41 & 0.79 & 0.32 & 0.84 \\
\hline & 2 & 0.81 (0.64 to 0.91) & $66\left(\times 10^{3} / \mu \mathrm{L}\right)$ & 0.81 & 0.79 & 0.48 & 0.94 \\
\hline \multirow[t]{2}{*}{ PT-INR } & Day 0 & 0.53 (0.34 to 0.72$)$ & 1.2 & 0.53 & 0.71 & 0.31 & 0.86 \\
\hline & 2 & 0.68 (0.47 to 0.84$)$ & 1.5 & 0.61 & 0.81 & 0.43 & 0.89 \\
\hline \multirow[t]{2}{*}{ FDP } & Day 0 & 0.61 (0.42 to 0.76$)$ & $21(\mu \mathrm{g} / \mathrm{mL})$ & 0.61 & 0.65 & 0.29 & 0.87 \\
\hline & 2 & 0.61 (0.41 to 0.77$)$ & $22(\mu \mathrm{g} / \mathrm{mL})$ & 0.67 & 0.65 & 0.31 & 0.89 \\
\hline \multicolumn{8}{|c|}{ Thrombin generation } \\
\hline \multirow[t]{2}{*}{ TAT } & Day 0 & 0.77 (0.62 to 0.87$)$ & $18(\mathrm{ng} / \mathrm{mL})$ & 0.81 & 0.77 & 0.46 & 0.94 \\
\hline & 2 & 0.83 (0.65 to 0.93$)$ & $16(\mathrm{ng} / \mathrm{mL})$ & 0.67 & 0.92 & 0.67 & 0.92 \\
\hline \multicolumn{8}{|c|}{ Anticoagulant activity } \\
\hline \multirow[t]{2}{*}{ PC } & Day 0 & 0.64 (0.45 to 0.79$)$ & $37(\%)$ & 0.53 & 0.75 & 0.35 & 0.87 \\
\hline & 2 & 0.76 (0.53 to 0.89$)$ & $22(\%)$ & 0.61 & 0.97 & 0.82 & 0.91 \\
\hline \multicolumn{8}{|c|}{ Fibrinolytic activity } \\
\hline \multirow[t]{2}{*}{ Plasminogen } & Day 0 & 0.64 (0.45 to 0.79$)$ & $61(\%)$ & 0.81 & 0.52 & 0.29 & 0.91 \\
\hline & 2 & 0.75 (0.57 to 0.87$)$ & $50(\%)$ & 0.81 & 0.67 & 0.38 & 0.93 \\
\hline \multirow[t]{2}{*}{ PAl-1 } & Day 0 & 0.81 (0.64 to 0.91$)$ & $269(\mathrm{ng} / \mathrm{mL})$ & 0.85 & 0.71 & 0.38 & 0.96 \\
\hline & 2 & 0.91 (0.79 to 0.96$)$ & 81.4 (ng/mL) & 0.69 & 0.97 & 0.82 & 0.94 \\
\hline
\end{tabular}

AUC, area under the curve; $\mathrm{Cl}$, confidence interval; FDP, fibrin degradation products; NPV, negative predictive value; PAl-1, plasminogen activator inhibitor-1; PC, protein C activity; PPV, positive predictive value; PT-INR, prothrombin time-international normalized ratio; ROC, receiver operating characteristic; TAT, thrombin-antithrombin complex.

${ }^{*}$ Cutoff values were calculated to maximize the sum of sensitivity and specificity.

fibrinolysis develop early in the course of sepsis, and these three mechanisms should be evaluated individually for the diagnosis of DIC in patients with sepsis.

In this study, we found that TAT, a marker of thrombin generation, and PAI-1, which is induced by pro-inflammatory cytokines, were highest at baseline and improved when diagnosis of DIC was made in patients



Figure 3 Kaplan-Meier survival curves for patients grouped by cutoff points of TAT and PAI-1 at baseline. The cutoff points were set at $18 \mathrm{ng} / \mathrm{mL}$ for thrombin-antithrombin complex (TAT) and $270 \mathrm{ng} / \mathrm{mL}$ for plasminogen activator inhibitor-1 (PAl-1), based on the best calculated cutoff values that maximize the sum of sensitivity and specificity for 28-day mortality. who developed overt DIC. These significant trends were obvious in survivors with overt DIC. In non-survivors with overt DIC, elevated levels of TAT and PAI-1 persisted during the study period. Similar trends in those biomarkers were observed in an experimental model of sepsis and in clinical studies [25,26]. TAT and PAI-1 have short half-lives and they are produced early in the course of septic coagulopathy, while other biomarkers, such as platelets, PT-INR or PC, are the markers of consumption. The differences in those biomarkers over time between survivors and non-survivors indicate that TAT and PAI-1 may well reflect disease progress in septic coagulopathy.

Current criteria for early diagnosis of DIC have some potential limitations. Considering easy implementation, most criteria, including ISTH non-overt DIC and JAAM acute DIC criteria, use readily available coagulation tests for scoring. However, it is clear that global coagulation tests, such as PT and platelet count, primarily reflect the result of consumption and impaired synthesis rather than direct ongoing coagulopathy. Kinasewitz et al. [27] and Dhainaut et al. [28] established a simple diagnostic scoring system for the acute phase of septic coagulopathy, but these systems depend partly on worsening trends of global markers, which take at least two days to identify. 
Several hemostatic molecular biomarkers, including AT, PC, TAT, PIC and PAI-1, have also been evaluated in patients with sepsis, but the reported results were inconsistent $[1,24,25,28-30]$. Several possible explanations could account for these conflicting results. First, we demonstrated dynamic changes in the biomarkers within a few days in the initial phase of sepsis, which is consistent with previous studies $[1,25]$; therefore, the timing of biomarker measurement is important for interpretation of the results. Second, the cutoff value is another factor that influences the diagnostic ability of biomarkers. Oh et al. [6] and Egi et al. [31] evaluated the cutoff value of the lower limit of normal (70\%) in AT levels for ISTH non-overt DIC criteria, and showed that the diagnostic ability for overt DIC did not improve by adding AT to non-overt DIC criteria. In our study, AT activity, as well as PC, was decreased below the lower limit of normal, even in most of the patients without overt DIC, and the cutoff value of AT level to discriminate patients with and without overt DIC was much lower (46.1\%). Last, most of the previous studies evaluated the impact of hemostatic biomarkers on prognosis in patients with sepsis. We found that some plasma biomarkers at baseline were good predictors for the development of overt DIC, but were less predictive for 28-day mortality compared with Day 2 markers, which indicates that persistence of coagulopathy, rather than just the development of it, influences the prognosis in patients with sepsis. In addition, multiple interactive systemic factors other than coagulopathy would be involved in the pathogenesis of organ failure and the risk of mortality. $\mathrm{We}$, therefore, evaluated diagnostic and prognostic values of biomarkers individually.

There were some potential limitations to our study. First, this was a prospective observational study conducted in a single center with a relatively small population size. Although the overall rate of DIC matched that in previous studies [3,32], our cohort included fewer pneumonia patients, who often die from respiratory failure rather than multiple organ failure, including DIC. A large validation study is needed to confirm our results. Second, there is no gold standard for diagnosis of or the criteria for intervention in sepsis-associated coagulopathy. We used the ISTH overt DIC criteria as the diagnostic standard, considering coagulopathy that fulfilled these criteria would be severe enough to be eligible for intervention. Third, although our management of sepsis followed the SSCG guidelines, and did not deviate from standard care, prophylactic anticoagulation and interventions, such as blood transfusion as well as AT substitution, may have influenced the levels of hemostatic biomarkers except for baseline profile, and their relationship with the scores of overt DIC. Last, our study lacked explanations about why TAT, PAI-1 and PC were the best diagnostic markers for overt DIC. We found a strong correlation among AT, PC, plasminogen and $\alpha_{2}$-PI at baseline. Considering that the same mechanism of consumption might be the main reason for decreased activity of those biomarkers [33], it is unclear why PC had superior diagnostic ability. Of particular interest is the contrast between the diagnostic value of TAT and another thrombin generation marker, SF. One possible explanation is the differences in half-life or mechanisms of clearance, where TAT has a shorter half-life (10 to 15 minutes), compared with SF (several hours). Further study is needed to better understand the processes of these biomarkers, and for the development of new therapeutic strategies in septic DIC.

\section{Conclusions}

The results of our study provide evidence that almost half of the patients developed severe coagulopathy in the initial phase of sepsis, which was demonstrated by baseline abnormalities in hemostatic biomarkers and their strong association with subsequent fulfillment of overt DIC criteria. In particular, a single determination of TAT, PAI-1 and PC activity at ICU admission allowed early identification of severe coagulopathy, or DIC, leading to early intervention for patients with sepsis.

\section{Key messages}

- The present study showed that coagulopathy was frequently observed in the initial phase of sepsis, and severe coagulation and fibrinolytic abnormalities were strongly associated with subsequent development of overt DIC.

- Among the 14 plasma biomarkers evaluated, TAT, PAI-1 and PC activity on ICU admission were the best combination to discriminate between patients with and without overt DIC.

- In terms of predicting mortality, only TAT and PAI-1 were significant predictors of 28-day mortality at the time of ICU admission.

\section{Additional files}

Additional file 1: Figure S1. Time course of biomarkers from baseline
to Day 3. Fibrinogen, soluble fibrin (SF), plasminogen, $a_{2}$-plasmin inhibitor
( $a$ 2-PI), plasmin- $a_{2}$-plasmin inhibitor complex (PIC) and soluble E-selectin
(sES) for patients with and without subsequent development of overt dis-
seminated intravascular coagulation (DIC) (gray vs. white bars), and for
survivors (dotted line) and non-survivors (solid line) among
patients with overt DIC. Data are expressed as mean and $95 \% \mathrm{Cl} .{ }^{*} P<0.05$
between patients with and without overt DIC on the same day. ${ }^{*} P<0.05$
between patients on Day 0 versus Day $2 .{ }^{+} P<0.05$ between survivors and
non-survivors with overt DIC on the same day.
Additional file 2: Figure S2. Correlation of plasma biomarkers at
baseline with each other. The correlation graphs and Spearman rank
correlation coefficients ( $r$ value) are shown here.
Additional file 3: Table S1. Area under ROC curves of Day 0 and Day 2
biomarkers for prediction of mortality.

Additional file 1: Figure S1. Time course of biomarkers from baseline to Day 3. Fibrinogen, soluble fibrin (SF), plasminogen, $a_{2}$-plasmin inhibitor (a2-PI), plasmin-a - plasmin inhibitor complex (PIC) and soluble E-selectin (SES) for patients with and without subsequent development of overt disseminated intravascular coagulation (DIC) (gray vs. white bars), and for survivors (dotted line) and non-survivors (solid line) among patients with overt DIC. Data are expressed as mean and 95\% Cl. ${ }^{*} \mathrm{P}<0.05$ between patients with and without overt DIC on the same day. ${ }^{* *} P<0.05$ between patients on Day 0 versus Day $2 .{ }^{+} P<0.05$ between survivors and non-survivors with overt DIC on the same day.

Additional file 2: Figure S2. Correlation of plasma biomarkers at baseline with each other. The correlation graphs and Spearman rank Additional file 3: Table S1. Area under ROC curves of Day 0 and Day 2 biomarkers for prediction of mortality. 


\section{Abbreviations}

$a_{2}$-PI: $a_{2}$-plasmin inhibitor; APACHE: Acute Physiology and Chronic Health Evaluation; AT: Antithrombin; AUROC: Area under the receiver operating curve; DIC: Disseminated intravascular coagulation; FDP: Fibrin degradation product; ISTH: International Society on Thrombosis and Haemostasis; JAAM: Japanese Association for Acute Medicine; NPV: Negative predictive value; PAl-1: Plasminogen activator inhibitor-1; PC: Protein C; PIC: Plasmin- $\mathrm{a}_{2}^{-}$ plasmin inhibitor complex; PPV: Positive predictive value; PT-INR: Prothrombin time-international normalized ratio; ROC: Receiver operating characteristic; sES: soluble E-selectin; SF: Soluble fibrin; SOFA: Sequential Organ Failure Assessment; TAT: Thrombin-antithrombin complex.

\section{Competing interests}

The authors declare that they have no competing interests.

\section{Authors' contributions}

KK conceived and designed the study. KK and SN prepared the data for analysis. KK conducted the data analysis. SM assisted with interpretation of the results. YS, JM and SN supervised the study. KK and SM drafted the article. All authors read and approved the manuscript. KK and SM take responsibility for the paper as a whole.

\section{Acknowledgements}

The authors thank the research staff of the Divisions of Cell and Molecular Medicine and the nursing staff of the Intensive Care Unit at Jichi Medical University Hospital for their assistance.

Received: 30 September 2013 Accepted: 6 January 2014

Published: 13 January 2014

\section{References}

1. Kinasewitz GT, Yan SB, Basson B, Comp P, Russell JA, Cariou A, Um SL, Utterback B, Laterre PF, Dhainaut JF, PROWESS Sepsis Study Group: Universal changes in biomarkers of coagulation and inflammation occur in patients with severe sepsis, regardless of causative micro-organism [ISRCTN74215569]. Crit Care 2004, 8:R82-R90.

2. Levi M, Ten Cate H: Disseminated intravascular coagulation. N Engl J Med 1999, 341:586-592.

3. Zeerleder S, Hack CE, Wuillemin WA: Disseminated intravascular coagulation in sepsis. Chest 2005, 128:2864-2875.

4. Taylor FB Jr, Toh CH, Hoots WK, Wada H, Levi M, Scientific Subcommittee on Disseminated Intravascular Coagulation (DIC) of the International Society on Thrombosis and Haemostasis (ISTH): Towards definition, clinical and laboratory criteria, and a scoring system for disseminated intravascular coagulation. Thromb Haemost 2001, 86:1327-1330.

5. Dhainaut JF, Yan SB, Joyce DE, Pettila V, Basson B, Brandt JT, Sundin DP, Levi M: Treatment effects of drotrecogin alfa (activated) in patients with severe sepsis with or without overt disseminated intravascular coagulation. J Thromb Haemost 2004, 2:1924-1933.

6. Oh D, Jang MJ, Lee SJ, Chong SY, Kang MS, Wada H: Evaluation of modified non-overt DIC criteria on the prediction of poor outcome in patients with sepsis. Thromb Res 2010, 126:18-23.

7. Angstwurm MW, Dempfle CE, Spannagl M: New disseminated intravascular coagulation score: a useful tool to predict mortality in comparison with Acute Physiology and Chronic Health Evaluation II and Logistic Organ Dysfunction scores. Crit Care Med 2006, 34:314-320. quiz 328.

8. Voves C, Wuillemin WA, Zeerleder S: International Society on Thrombosis and Haemostasis score for overt disseminated intravascular coagulation predicts organ dysfunction and fatality in sepsis patients. Blood Coagul Fibrinolysis 2006, 17:445-451.

9. Ten Cate H: Trombocytopenia: one of the markers of disseminated intravascular coagulation. Pathophysiol Haemost Thromb 2003, 33:413-416.

10. Song J, Hu D, He C, Wang T, Liu X, Ma L, Lin Z, Chen Z: Novel biomarkers for early prediction of sepsis-induced disseminated intravascular coagulation in a mouse cecal ligation and puncture model. I Inflamm (Lond) 2013, 10:7.

11. Toh CH, Downey C: Performance and prognostic importance of a new clinical and laboratory scoring system for identifying non-overt disseminated intravascular coagulation. Blood Coagul Fibrinolysis 2005, 16:69-74.

12. Levi M, van der Poll T: Inflammation and coagulation. Crit Care Med 2010, 38:S26-S34.

13. O'Brien M: The reciprocal relationship between inflammation and coagulation. Top Companion Anim Med 2012, 27:46-52.

14. Koyama K, Madoiwa S, Tanaka S, Koinuma T, Wada M, Sakata A, Ohmori T, Mimuro J, Nunomiya S, Sakata Y: Evaluation of hemostatic biomarker abnormalities that precede platelet count decline in critically ill patients with sepsis. J Crit Care 2013, 28:556-563.

15. Levy MM, Fink MP, Marshall JC, Abraham E, Angus D, Cook D, Cohen J, Opal SM, Vincent JL, Ramsay G, International Sepsis Definitions Conference: SCCM/ESICM/ACCP/ATS/SIS International Sepsis Definitions Conference. Intensive Care Med 2001, 2003(29):530-538.

16. Knaus WA, Draper EA, Wagner DP, Zimmerman JE: APACHE II: a severity of disease classification system. Crit Care Med 1985, 13:818-829.

17. Vincent JL, Moreno R, Takala J, Willatts S, De Mendonca A, Bruining H, Reinhart CK, Suter PM, Thijs LG: The SOFA (Sepsis-related Organ Failure Assessment) score to describe organ dysfunction/failure. On behalf of the Working Group on Sepsis-Related Problems of the European Society of Intensive Care Medicine. Intensive Care Med 1996, 22:707-710

18. Gando S, Iba T, Eguchi Y, Ohtomo Y, Okamoto K, Koseki K, Mayumi T, Murata A, Ikeda T, Ishikura H, Ueyama M, Ogura H, Kushimoto S, Saitoh D, Endo S, Shimazaki S, Japanese Association for Acute Medicine Disseminated Intravascular Coagulation (JAAM DIC) Study Group: A multicenter, prospective validation of disseminated intravascular coagulation diagnostic criteria for critically ill patients: comparing current criteria. Crit Care Med 2006, 34:625-631.

19. Dellinger RP, Levy MM, Carlet JM, Bion J, Parker MM, Jaeschke R, Reinhart K, Angus DC, Brun-Buisson C, Beale R, Calandra T, Dhainaut JF, Gerlach H, Harvey M, Marini JJ, Marshall J, Ranieri M, Ramsay G, Sevransky J, Thompson BT, Townsend S, Vender JS, Zimmerman JL, Vincent JL: Surviving Sepsis Campaign: international guidelines for management of severe sepsis and septic shock: 2008. Intensive Care Med 2008, 34:17-60. Erratum in: Intensive Care Med 2008, 34:783-785.

20. Amaral A, Opal SM, Vincent JL: Coagulation in sepsis. Intensive Care Med 2004, 30:1032-1040.

21. Faust SN, Levin M, Harrison OB, Goldin RD, Lockhart MS, Kondaveeti S, Laszik Z, Esmon CT, Heyderman RS: Dysfunction of endothelial protein C activation in severe meningococcal sepsis. N Engl J Med 2001, 345:408-416.

22. Levi $\mathrm{M}$, van der Poll $\mathrm{T}$ : The role of natural anticoagulants in the pathogenesis and management of systemic activation of coagulation and inflammation in critically ill patients. Semin Thromb Hemost 2008, 34:459-468.

23. Bergmann S, Hammerschmidt S: Fibrinolysis and host response in bacterial infections. Thromb Haemost 2007, 98:512-520.

24. Madoiwa S, Nunomiya S, Ono T, Shintani Y, Ohmori T, Mimuro J, Sakata Y: Plasminogen activator inhibitor 1 promotes a poor prognosis in sepsis-induced disseminated intravascular coagulation. Int I Hematol 2006, 84:398-405.

25. Lorente JA, Garcia-Frade L, Landin L, de Pablo R, Torrado C, Renes E, Garcia-Avello A: Time course of hemostatic abnormalities in sepsis and its relation to outcome. Chest 1993, 103:1536-1542.

26. Saetre T, Lindgaard AK, Lyberg T: Systemic activation of coagulation and fibrynolysis in a porcine model of serogroup A streptococcal shock. Blood Coagul Fibrinolysis 2000, 11:433-438.

27. Kinasewitz GT, Zein JG, Lee GL, Nazir SA, Taylor FB Jr: Prognostic value of a simple evolving disseminated intravascular coagulation score in patients with severe sepsis. Crit Care Med 2005, 33:2214-2221.

28. Dhainaut JF, Shorr AF, Macias WL, Kollef MJ, Levi M, Reinhart K, Nelson DR: Dynamic evolution of coagulopathy in the first day of severe sepsis: relationship with mortality and organ failure. Crit Care Med 2005, 33:341-348.

29. Iba T, Gando S, Murata A, Kushimoto S, Saitoh D, Eguchi Y, Ohtomo Y, Okamoto K, Koseki K, Mayumi T, Ikeda T, Ishhikura H, Ueyama M, Ogura Y, Endo S, Shimazaki S, Japanese Association for Acute Medicine Disseminated Intravascular Coagulation Study Group: Predicting the severity of systemic inflammatory response syndrome (SIRS)-associated coagulopathy with hemostatic molecular markers and vascular endothelial injury markers. J Trauma 2007, 63:1093-1098. 
30. Iba T, Kidokoro A, Fukunaga M, Sugiyama K, Sawada T, Kato H: Association between the severity of sepsis and the changes in hemostatic molecular markers and vascular endothelial damage markers. Shock 2005, 23:25-29.

31. Egi M, Morimatsu H, Wiedermann CJ, Tani M, Kanazawa T, Suzuki S, Matsusaki T, Shimizu K, Toda Y, Iwasaki T, Morita K: Non-overt disseminated intravascular coagulation scoring for critically ill patients: the impact of antithrombin levels. Thromb Haemost 2009, 101:696-705.

32. Kienast J, Juers M, Wiedermann CJ, Hoffmann JN, Ostermann H, Strauss R, Keinecke HO, Warren BL, Opal SM, KyberSept Investigators: Treatment effects of high-dose antithrombin without concomitant heparin in patients with severe sepsis with or without disseminated intravascular coagulation. J Thromb Haemost 2006, 4:90-97.

33. Mavrommatis AC, Theodoridis T, Economou M, Kotanidou A, El Ali M, Christopoulou-Kokkinou V, Zakynthinos SG: Activation of the fibrinolytic system and utilization of the coagulation inhibitors in sepsis: comparison with severe sepsis and septic shock. Intensive Care Med 2001, 27:1853-1859.

doi: $10.1186 /$ cc13190

Cite this article as: Koyama et al:: Combination of thrombin-antithrombin complex, plasminogen activator inhibitor-1, and protein $C$ activity for early identification of severe coagulopathy in initial phase of sepsis: a prospective observational study. Critical Care 2014 18:R13.

\section{Submit your next manuscript to BioMed Central and take full advantage of:}

- Convenient online submission

- Thorough peer review

- No space constraints or color figure charges

- Immediate publication on acceptance

- Inclusion in PubMed, CAS, Scopus and Google Scholar

- Research which is freely available for redistribution 